\title{
Origin of Hawking radiation: firewall or atmosphere?
}

\author{
Wontae $\mathrm{Kim}^{1, *}$ \\ ${ }^{1}$ Department of Physics, Sogang University, Seoul 04107, South Korea
}

(Dated: January 17, 2017)

\begin{abstract}
The Unruh vacuum not admitting any outgoing flux at the horizon implies that the origin of the outgoing Hawking radiation is the atmosphere of a near-horizon quantum region without resort to the firewall; however, the existence of the firewall of superplanckian excitations at the horizon can be supported by the infinite Tolman temperature at the horizon. In an exactly soluble model, we explicitly show that the firewall necessarily emerges out of the Unruh vacuum so that the Tolman temperature in the Unruh vacuum is divergent in essence due to the infinitely blueshifted negative ingoing flux crossing the horizon rather than the outgoing flux. We also show that the outgoing Hawking radiation in the Unruh vacuum indeed originates from the atmosphere, not just at the horizon, which is of no relevance to the infinite blueshift. Consequently, the firewall from the infinite Tolman temperature and the Hawking radiation from the atmosphere turn out to be compatible, once we waive the claim that the Hawking radiation in the Unruh vacuum originates from the infinitely blueshifted outgoing excitations at the horizon.
\end{abstract}

Keywords: Boulware vacuum, Israel-Hartle-Hawking vacuum, Unruh vacuum, Tolman temperature, StefanBoltzmann law, Firewall

\footnotetext{
*wtkim@sogang.ac.kr
} 


\section{INTRODUCTION}

Hawking radiation as an information carrier [1] is subject to observers, which requires black hole complementarity such that there are no contradictory physical observations between different observers [2]. Recently, the firewall paradox [3] (or the energetic curtain from some different assumptions [4]) was that the assumptions of the purity of the Hawking radiation, the semiclassical quantum field theory, and the equivalence principle are not consistent with one another. Then they suggested a conservative solution to this paradox that the infalling observer when crossing the horizon could find the firewall of high frequency quanta beyond the Planckian scale after the Page time [5]. Subsequently, there have been many arguments for this paradox and alternatives [6-13].

On the other hand, Unruh attained a startling conclusion that Hawking radiation appears in the absence of the outgoing flux at the horizon [14] and also showed that the process of thermal particle creation is low energy behavior and the highest frequency mode does not matter for the thermal emission by using a modification of the dispersion relation in a sonic black hole numerically [15]. Moreover, it was shown that the effective blueshift of the outgoing Hawking radiation remains finite by averaging out the Tolman factor outside the horizon in terms of the Poisson distribution [16]. All these imply that the Hawking radiation originates from a macroscopic distance outside the horizon. Interestingly, it was also claimed that the Hawking radiation can be retrieved by an alternative scenario that a positive outward flux at the horizon is interpreted as the negative influx without recourse to a pair creation scenario [17].

Recently, there was a refined question concerning the origin of the Hawking radiation in the Unruh vacuum by Giddings [18], where the evidence concerns the three relevant points that are: (1) the effective emitting area of Hawking radiation is considerably larger than the size defined by the area of the black hole [19], (2) there is no outgoing flux at the horizon in the Unruh vacuum and there should appear a transition from the ingoing to the outgoing flux over a large quantum region, and (3) the size of wave length of a thermal Hawking particle is larger than the horizon size. One of the essential ingredients is that the transition from the ingoing to the outgoing flux should appear over the atmosphere of the quantum region outside the horizon without resort to the firewall. Moreover, the importance of the atmosphere was also emphasized in connection with the nonviolent scenarios for the 
information loss paradox [20].

However, the existence of the firewall seems obvious from the fact that the Tolman temperature defined in the Unruh vacuum is infinite on the horizon [21], which is indeed due to the infinite blueshift of the Hawking temperature there. In other words, the thermal Hawking particles at infinity might be ascribed to the infinitely blueshifted outgoing radiation at the horizon or very near the horizon. This argument, as mentioned above, would not be reliable, since the Unruh vacuum does not admit any outgoing flux on the horizon semiclassically [14]. So it is not likely to get the firewall from the outgoing flux, and thus it might be tempting to conclude that the firewall is incompatible with the Unruh vacuum. In these regards, the origin of Hawking radiation and the reason for the existence of the firewall as well as their relationship still seem to be equivocal in spite of many efforts.

In this work, we elucidate how the Hawking radiation and the firewall appear simultaneously in a tractable field theoretic model, and then provide a compelling argument for their compatibility between the firewall and the Hawking radiation. The key is to decompose quantitatively the Tolman temperature read off from the Stefan-Boltzmann law into the two chiral temperatures of $T_{\mathrm{L}}, T_{\mathrm{R}}$ defined by the negative influx and the positive outward flux, respectively, and then identify their properties carefully. It will be shown that $T_{\mathrm{L}}$ becomes infinite at the horizon, which is regarded as a signal of the firewall; however, it vanishes at infinity, so that it does not affect the asymptotic observer at infinity. The essential reason for the existence of the firewall is due to the infinitely blueshifted negative influx crossing the horizon rather than the outward flux. On the other hand, $T_{R}$ will be shown to be finite everywhere by identifying it with a newly derived effective Tolman temperature from the modified Stefan-Boltzmann law. In particular, it vanishes at the horizon and approaches the Hawking temperature at infinity. Thus, it shows that the outgoing Hawking radiation originates from the atmosphere of the near-horizon quantum region, not just at the horizon. After all, the present analysis in the semiclassically fixed background approximation will show that the firewall is not only a conservative but also a natural solution in the Unruh vacuum, and the Hawking radiation indeed originates from the atmosphere without any conflicts with the firewall.

The organization of this paper is as follows. In Sec. II, we introduce the quantized stress tensor for a single scalar field on a general class of two-dimensional black hole background without choosing a specific metric, and discuss black-hole vacua. In Sec. III, in the Israel- 
Hartle-Hawking vacuum [22, 23], we recapitulate the recent formulation of the effective Tolman temperature [24] and then write it in terms of a more preferable form in order to serve our purpose. In thermal equilibrium, the effective Tolman temperature turns out to be a natural generalization of the usual Tolman temperature in the presence of conformal anomaly [25] responsible for the Hawking radiation [26]. In Sec. IV, the Tolman temperature defined in the Unruh vacuum is decomposed into the left and right temperatures from the Stefan-Boltzmann law for a radiating system by using the results of the preceding sections. Then the arguments for the firewall and the atmosphere are explicitly discussed by choosing a specific metric. Finally, conclusion and discussion are given in Sec. V.

\section{ENERGY DENSITY AND FLUX}

Let us start with a two-dimensional general static black hole described by the metric,

$$
d s^{2}=-g(r) d t^{2}+\frac{1}{g(r)} d r^{2}
$$

where $g(r)$ is an asymptotically flat metric function. The constants are set to $\hbar=k_{\mathrm{B}}=$ $G=c=1$. The event horizon $r_{\mathrm{H}}$ is defined by $g\left(r_{\mathrm{H}}\right)=0$, and the Hawking temperature is calculated from the definition of the surface gravity as $T_{\mathrm{H}}=g^{\prime}\left(r_{\mathrm{H}}\right) / 4 \pi[1]$ where the prime denotes the derivative with respect to $r$. The Hawking temperature is blueshifted for a distant observer outside the horizon [27], which is simply written as the Tolman form [21].

From the covariant conservation law and the conformal anomaly of $\left\langle T_{\mu}^{\mu}\right\rangle=R /(24 \pi)$ for a two-dimensional massless scalar field [25], the components of the stress tensor are determined as $\left\langle T_{ \pm \pm}\right\rangle=\left(g g^{\prime \prime}-(1 / 2) g^{2}+t_{ \pm}\right) /(96 \pi),\left\langle T_{+-}\right\rangle=g g^{\prime \prime} /(96 \pi)$, where $t_{ \pm}$reflect the non-locality of the conformal anomaly [26]. Note that the expectation value of the energy-momentum tensor was written by using the tortoise coordinates written in the form of light-cone where $\sigma^{ \pm}=t \pm r^{*}(r)$ and $r^{*}=\int d r / g(r)$, but $g(r)$ was just written in terms of $r$ instead of $r^{*}$ for convenience [18]. Then, the proper energy density, pressure, and flux can be defined as $\varepsilon=\left\langle T_{\mu \nu}\right\rangle u^{\mu} u^{\nu}, p=\left\langle T_{\mu \nu}\right\rangle n^{\mu} n^{\nu}$, and $\mathcal{F}=-\left\langle T_{\mu \nu}\right\rangle u^{\mu} n^{\nu}$, where $u^{\mu}$ is a two-velocity and $n^{\mu}$ is a spacelike unit normal vector satisfying $n^{\mu} n_{\mu}=1$ and $n^{\mu} u_{\mu}=0$. Explicitly, in the light-cone coordinates, the velocity vector from the geodesic equation of motion and the normal vector are solved in a freely falling frame from rest as [28]

$$
u^{+}=u^{-}=n^{+}=-n^{-}=\frac{1}{\sqrt{g}} .
$$


In particular, the proper energy density and flux are expressed by

$$
\begin{aligned}
\varepsilon & =\frac{1}{g}\left(\left\langle T_{++}\right\rangle+\left\langle T_{--}\right\rangle+2\left\langle T_{+-}\right\rangle\right), \\
\mathcal{F} & =-\frac{1}{g}\left(\left\langle T_{++}\right\rangle-\left\langle T_{--}\right\rangle\right),
\end{aligned}
$$

where the redundant pressure is related to the energy density via the trace relation of $\left\langle T_{\mu}^{\mu}\right\rangle=-\varepsilon+p$. From Eqs. (3) and (4), the energy density and flux are explicitly written as $\varepsilon=\left(4 g g^{\prime \prime}-g^{2}+t_{+}+t_{-}\right) /(96 \pi g)$ and $\mathcal{F}=-\left(t_{+}-t_{-}\right) /(96 \pi g)$.

In the Israel-Hartle-Hawking vacuum [22, 23], the stress tensor is regular at both the future horizon and the past horizon, so that the regularity condition determines the integration constants as $t_{+}=t_{-}=(1 / 2) g^{2}\left(r_{\mathrm{H}}\right)$. Let us assume that the metric function is finite at least up to the second derivative with $g^{\prime \prime}<0$, for instance, which holds for the Schwarzschild black hole or the CGHS black hole [29], then the curvature scalar of $R=-g^{\prime \prime}$ is positive finite. The proper energy density (3) also becomes finite everywhere. In particular, it is negative finite at the horizon, $\varepsilon_{\mathrm{HH}}\left(r_{\mathrm{H}}\right)=g^{\prime \prime}\left(r_{\mathrm{H}}\right) /(48 \pi)$, while it is positive finite at infinity, $\varepsilon_{\mathrm{HH}}(\infty)=g^{\prime 2}\left(r_{\mathrm{H}}\right) /(96 \pi)$. It shows that the proper energy density is not always positive.

There is another equilibrium state defined by $t_{+}=t_{-}=0$ called the Boulware vacuum [30]. The energy density (3) is negatively divergent at the horizon, $\varepsilon_{\mathrm{B}}\left(r_{\mathrm{H}}\right) \rightarrow-\infty$ and negatively vanishes at infinity, $\varepsilon_{\mathrm{B}}(\infty)=0$. If such a black hole exists, then it will be surrounded by the negative energy density in equilibrium. Note that the energy density is divergent at the horizon, so that the smoothness of the horizon is not warranted.

The Unruh vacuum of our interest is considered by twisting two equilibrium states asymmetrically in such a way that $t_{+}=0$ and $t_{-}=g^{\prime 2}\left(r_{\mathrm{H}}\right) / 2$ in order to describe an evaporating black hole semiclassically [14]. Then the proper energy density is negative infinity at the horizon, $\varepsilon_{\mathrm{U}}\left(r_{\mathrm{H}}\right) \rightarrow-\infty$ like the case of the Boulware vacuum defined by $t_{+}=t_{-}=0$ [30]. It is positive finite at infinity like the case of the Israel-Hartle-Hawking vacuum defined by $t_{+}=t_{-}=(1 / 2) g^{\prime 2}\left(r_{\mathrm{H}}\right)[22,23]$, but its magnitude is half of that of the Israel-Hartle-Hawking vacuum, i.e., $\varepsilon_{\mathrm{U}}(\infty)=g^{\prime 2}\left(r_{\mathrm{H}}\right) /(192 \pi)=\varepsilon_{\mathrm{HH}}(\infty) / 2$. As expected, the non-vanishing flux is obtained as $\mathcal{F}_{\mathrm{U}}(r)=g^{\prime 2}(r) /(192 \pi g)$ which is coincident with the energy density at infinity, but it is divergent at the horizon.

Note that the above expectation value of the energy-momentum tensor $\left\langle T_{\mu \nu}\right\rangle$ in any vacua is finite at the horizon, whereas the proper flux and the energy density in the Unruh vacuum are divergent there. One might wonder what the origin of the divergence is. The expectation 
value of the energy-momentum tensor $\left\langle T_{a b}\left(\xi^{a}, \xi^{b}\right)\right\rangle$ defined in a locally inertial coordinate system can be obtained from the general coordinate transformation of $\left\langle T_{\mu \nu}\right\rangle$ defined in the tortoise coordinate system of $\sigma^{ \pm}=t \pm r^{*}(r)$, which is implemented by

$$
\left\langle T_{a b}\right\rangle=\frac{\partial \sigma^{\mu}}{\partial \xi^{a}} \frac{\partial \sigma^{\nu}}{\partial \xi^{b}}\left\langle T_{\mu \nu}\right\rangle
$$

where $a, b=0,1$ are the indices for the locally inertial coordinate system and $\mu, \nu= \pm$ are for the tortoise coordinate system. We also consider the coordinate transformation of the velocity vector and the unit normal spacelike vector as

$$
u^{\mu}=\frac{\partial \sigma^{\mu}}{\partial \xi^{a}} u^{a}=\frac{\partial \sigma^{\mu}}{\partial \xi^{0}}, \quad n^{\mu}=\frac{\partial \sigma^{\mu}}{\partial \xi^{a}} n^{a}=\frac{\partial \sigma^{\mu}}{\partial \xi^{1}}
$$

where $u^{a}=(1,0), n^{a}=(0,1)$ are defined in the locally inertial coordinate system. Therefore, the flux $\mathcal{F}=-\left\langle T_{01}\right\rangle$ from Eq. (5) is written as

$$
-\left\langle T_{01}\right\rangle=-\frac{\partial \sigma^{\mu}}{\partial \xi^{0}} \frac{\partial \sigma^{\nu}}{\partial \xi^{1}}\left\langle T_{\mu \nu}\right\rangle=-u^{\mu} n^{\nu}\left\langle T_{\mu \nu}\right\rangle,
$$

by using Eqs. (6), which is nothing but Eq. (4) when Eq.(2) is used. The energy density (3) can also be obtained from the coordinate transformation of $\left\langle T_{00}\right\rangle=\left(\partial \sigma^{\mu} / \partial \xi^{0}\right)\left(\partial \sigma^{\nu} / \partial \xi^{0}\right)\left\langle T_{\mu \nu}\right\rangle=$ $u^{\mu} u^{\nu}\left\langle T_{\mu \nu}\right\rangle$, which reproduces Eq. (3). As a result, in the Unruh vacuum, the divergent proper flux at the horizon is due to the singular coordinate transformation at the horizon.

\section{STEFAN-BOLTZMANN LAW IN EQUILIBRIUM}

Conventionally, the Stefan-Boltzmann law in thermal equilibrium rests upon the traceless condition of the stress tensor [21]; however, it is worth noting that the trace anomaly is responsible for the Hawking radiation [26]. In that sense, assuming the nontrivial trace of the stress tensor, one should obtain a modified Stefan-Boltzmann law which gives an effective Tolman temperature induced by the trace anomaly [24]. Let us now obtain the effective Tolman temperature from the modified Stefan-Boltzmann law in the Israel-HartleHawking vacuum prior to the discussion in the case of the Unruh vacuum, and then write the effective temperature in terms of a much more convenient form for our purpose.

In a proper frame, the first law of thermodynamics reads as $d U=T d S-p d V$, where $U, T$, $S$, and $V$ are thermodynamic internal energy, temperature, entropy, and volume of a system in thermal equilibrium. Inserting the internal energy defined as $U=\int \varepsilon d V$ and the Maxwell 
relation of $(\partial S / \partial V)_{T}=(\partial p / \partial T)_{V}$ into the differentiated form of the thermodynamic first law with respect to the volume, one can obtain

$$
T\left(\frac{\partial \varepsilon}{\partial T}\right)_{V}-2 \varepsilon=\left\langle T_{\mu}^{\mu}\right\rangle
$$

where the trace anomaly is independent of the temperature [31]. From Eq. (8), the energy density is immediately solved as

$$
\varepsilon=\gamma T_{\mathrm{eff}}^{2}-\frac{1}{2}\left\langle T_{\mu}^{\mu}\right\rangle
$$

where $T_{\text {eff }}$ is the effective Tolman temperature. The integration constant $\gamma$ in Eq. (8) is the Stefan-Boltzmann constant given as $\gamma=\pi / 6$ for a massless scalar field [26]. The modified Stefan-Boltzmann law (9) simply reduces to the usual Stefan-Boltzmann law of $\varepsilon=\gamma T^{2}$ for the traceless case, which yields the usual Tolman temperature.

Note that the proper energy density in the Israel-Hartle-Hawking vacuum is not always positive [32]. Thus, one can find the position $r_{0}$ where the energy density vanishes, so that the region of the positive energy density is separated from that of the negative energy density. For example, the position is estimated as $r_{0} \sim 2.98 M$ in the two-dimensional Schwarzschild black hole [28]. It should be noted that the usual Stefan-Boltzmann law holds only at infinity such as $\varepsilon_{\mathrm{HH}}(\infty)=\gamma T_{\mathrm{H}}^{2}$; however, it does not appear to be reliable extremely in the region of the negative energy density for $r_{\mathrm{H}}<r<r_{0}$. Fortunately, the energy density need not be positive thanks to the anomalous term in the modified Stefan-Boltzmann law (9).

Now, plugging the expression for the energy density in Eq. (3) into Eq. (9), one can get the effective Tolman temperature in the Israel-Hartle-Hawking vacuum as

$$
\gamma T_{\mathrm{eff}}^{2}=\frac{1}{g}\left(\left\langle T_{++}\right\rangle_{\mathrm{HH}}+\left\langle T_{--}\right\rangle_{\mathrm{HH}}\right)=\frac{1}{96 \pi g}\left(2 g g^{\prime \prime}-g^{\prime 2}+g^{\prime 2}\left(r_{\mathrm{H}}\right)\right)
$$

where the stress tensors are calculated with respect to the Israel-Hartle-Hawking vacuum. In contrast to the divergent usual Tolman temperature at the horizon, the effective Tolman temperature (10) vanishes at the horizon, which can be shown by taking the limit at the horizon. In fact, there is neither influx nor outward flux at the horizon, and; thus, there is no reason for the firewall to exist. This fact is consistent with the regularity of the renormalized stress tensor at the horizon in the Israel-Hartle-Hawking vacuum [32] and the explicit calculation to make use of the detector [33]. 


\section{STEFAN-BOLTZMANN LAW IN A RADIATING SYSTEM}

Let us now derive the temperature for a radiating system such as the black hole in the Unruh vacuum. The temperature is obtained from the radiated power which is just the proper flux. The proper temperature in the Unruh vacuum can be read off from the twodimensional Stefan-Boltzmann law given by Giddings [18],

$$
\sigma T^{2}=-\frac{1}{g}\left(\left\langle T_{++}\right\rangle_{\mathrm{U}}-\left\langle T_{--}\right\rangle_{\mathrm{U}}\right)=\frac{\pi}{12}\left(\frac{T_{\mathrm{H}}}{\sqrt{g}}\right)^{2},
$$

where the stress tensors are calculated with respect to the Unruh vacuum. The twodimensional Stefan-Boltzmann constant in a radiating system $\sigma$ is half of that of the equilibrium state, so that $\sigma=\gamma / 2=\pi / 12$ is consistent. Note that the ingoing and outgoing stress tensors in the Unruh vacuum are the same as those in the Boulware and Israel-HartleHawking vacua, respectively. So the black hole temperature in the Unruh vacuum (11) can be decomposed into the left and right chiral temperatures without mixing chirality as

$$
\sigma T_{\mathrm{L}}^{2}=-\frac{\left\langle T_{++}\right\rangle_{\mathrm{B}}}{g}, \quad \sigma T_{\mathrm{R}}^{2}=\frac{\left\langle T_{--}\right\rangle_{\mathrm{HH}}}{g}
$$

where the stress tensors of $\left\langle T_{++}\right\rangle_{\mathrm{B}}$ and $\left\langle T_{--}\right\rangle_{\mathrm{HH}}$ are defined with respect to the Boulware and Israel-Hartle-Hawking vacua, respectively.

Equilibrium states of black holes are commonly described by the Boulware and IsraelHartle-Hawking vacua. This is possible only when the systems are locally in equilibrium and sufficiently slowly varying. In contrast to these vacua, the net flux is not zero for the Unruh vacuum, so that the black hole in this state is not in equilibrium as seen from Eq. (11). If the two equilibrium systems are interacting, then the thermal temperature for a better interpretation will require a quasi-equilibrium condition between the two different equilibrium systems. However, in the present semiclassical approximation, the influx and outward flux are actually decoupled and do not interfere with the influx and outward flux.

For the left temperature in Eq. (12), the ingoing flux is negative finite at the horizon as $\left\langle T_{++}\left(r_{\mathrm{H}}\right)\right\rangle_{\mathrm{B}}=-g^{\prime 2}\left(r_{\mathrm{H}}\right) /(192 \pi)$, so that the left temperature becomes positively divergent at the horizon as $T_{\mathrm{L}}\left(r_{H}\right) \rightarrow+\infty$. So the firewall in the Unruh vacuum arises from the infinite blueshift of the negative ingoing flux despite the absence of the outgoing flux at the horizon, so that the Tolman temperature is consequently divergent at the horizon. However, this ingoing flux decreases to zero and does not reach infinity, so that $T_{\mathrm{L}}(\infty)=0$. It implies 
that the ingoing superplanckian excitations have no impact on the asymptotic observer at infinity. Thus, these excitations are certainly responsible for the firewall but completely decoupled from the Hawking radiation at infinity.

Next, after rescaling $\sigma=\gamma / 2$, the right temperature in Eq. (12) can be shown to be equivalent to the effective Tolman temperature (10),

$$
T_{\mathrm{R}}=T_{\text {eff }},
$$

by use of the equilibrium condition of $\left\langle T_{++}\right\rangle_{\mathrm{HH}}=\left\langle T_{--}\right\rangle_{\mathrm{HH}}$. Thus, the right temperature directly possesses the same properties as those of the effective Tolman temperature, so that it is finite everywhere. In particular, it vanishes at the horizon and approaches the Hawking temperature exactly at the asymptotic infinity. Therefore, it shows that the Hawking particles at infinity originate from the atmosphere outside the horizon rather than the firewall.

On the other hand, from the left temperature in Eq. (12), it is of interest to note that one can also define a black hole temperature in the Boulware vacuum in a manner similar to the way of the Israel-Hartle-Hawking vacuum by replacing $\sigma=\gamma / 2$ and $\left\langle T_{++}\right\rangle_{\mathrm{B}}=$ $\left(\left\langle T_{++}\right\rangle_{\mathrm{B}}+\left\langle T_{--}\right\rangle_{\mathrm{B}}\right) / 2$ since $\left\langle T_{++}\right\rangle_{\mathrm{B}}=\left\langle T_{--}\right\rangle_{\mathrm{B}}$. So, the left-right temperatures in Eq. (12) can be compactly written in the unified manner as

$$
\gamma T_{\mathrm{L}, \mathrm{R}}^{2}=\mp \frac{1}{g}\left(\left\langle T_{++}\right\rangle_{\mathrm{B}, \mathrm{HH}}+\left\langle T_{--}\right\rangle_{\mathrm{B}, \mathrm{HH}}\right),
$$

where the local Boulware temperature and the local Hawking temperature are eventually on an equal footing, and thus, the ingoing particles are also in thermal states like the outgoing particles. It implies that the particles in the same chirality can be entangled with their partners, but the particles in a different chirality need not be entangled since they are not created from pair creation [17]. Importantly, this would be one of the advantages of the present analysis without recourse to pair creation, which could respect the monogamy principle in quantum mechanics.

So far, the origin of the Hawking radiation and the reason for the existence of the firewall have been discussed based on the generic metric. Let us now discuss the arguments explicitly in the two-dimensional Schwarzschild black hole described by $g(r)=1-2 M / r$. From Eq. (14), one can obtain the left and right temperatures as

$$
T_{\mathrm{L}}=\frac{1}{\sqrt{2} \pi r} \sqrt{\frac{M}{r-2 M}\left(1-\frac{3 M}{2 r}\right)},
$$




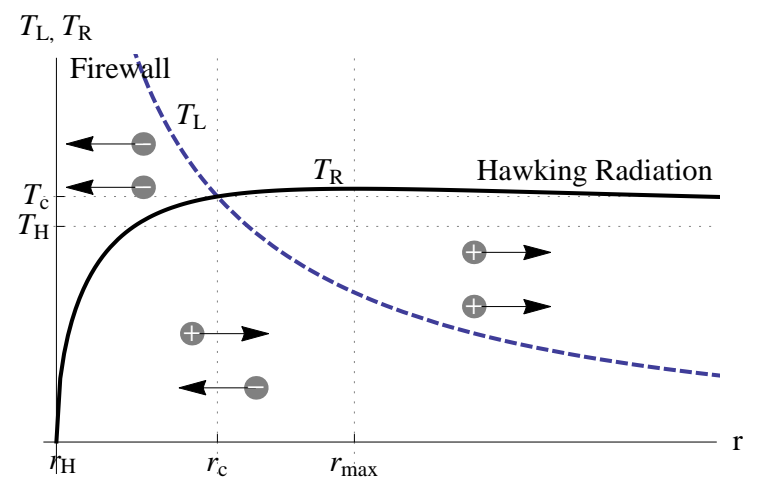

FIG. 1. The dashed curve is for $T_{\mathrm{L}}$, and the solid one is for $T_{\mathrm{R}}$, where $M=1$ for simplicity. The minus-plus signs in the small circles with the left and right arrows mean the negative influx and the positive outward flux, respectively. The critical position of the flux transition occurs at $r_{\mathrm{c}} \sim 3.26 M$ and the corresponding critical temperature $T_{\mathrm{c}}$ is slightly higher than the value of the Hawking temperature $T_{\mathrm{H}}$, where $T_{c} / T_{\mathrm{H}} \sim 1.14$. The maximum of the right temperature occurs at $r_{\max } \sim 4.32 M$.

$$
T_{\mathrm{R}}=\frac{1}{8 \pi M} \sqrt{1+\frac{2 M}{r}+\left(\frac{2 M}{r}\right)^{2}-3\left(\frac{2 M}{r}\right)^{3}} .
$$

Note that the left temperature is found to be infinite at the horizon and vanishes at infinity, which means that the firewall appears at the horizon and the asymptotic observer is free from the impact of the firewall. On the other hand, the right temperature is finite everywhere, and it shows that the outgoing radiation is a very low energy phenomenon since it is almost comparable to the Hawking temperature over the entire region outside the horizon. In particular, it vanishes at the horizon and approaches the Hawking temperature at infinity. As a corollary, from Eqs. (15) and (16) the Tolman temperature is obtained as $T=\sqrt{T_{\mathrm{L}}^{2}+T_{\mathrm{R}}^{2}}$ which shows only the collective behavior of the two chiral temperatures.

In Fig. 1, one may define a critical position $r_{c}$ at which the two excitation energies are the same as $T_{\mathrm{L}}=T_{\mathrm{R}}$. It yields $r_{\mathrm{c}} \sim 3.26 M$ which is a larger macroscopic distance as compared to the horizon size. For $r<r_{\mathrm{c}}$, the left temperature is dominant and eventually predicts the firewall at the horizon, whereas the right temperature is dominant for $r>r_{\mathrm{c}}$ and reproduces the Hawking temperature at infinity. Moreover, $T_{\mathrm{R}}$ has a peak at $r_{\max } \sim 4.32 \mathrm{M}$ which is larger than $r_{\mathrm{c}}$. Thus, the critical transition from the influx to the outward flux occurs before the right temperature arrives at the peak. 


\section{CONCLUSION AND DISCUSSION}

In conclusion, a glimpse of the Tolman temperature in the Unruh vacuum would lead to the conclusion that Hawking radiation at infinity comes from the infinitely blueshifted outgoing Hawking excitations at the horizon; however, it was a misleading interpretation due to the two overlapped features. In the context of the stress tensor calculations in this work, this issue was clarified by decomposing the Tolman temperature in the Unruh vacuum into the left and right chiral temperatures. We showed that the firewall in the Unruh vacuum comes from the negative influx crossing the horizon rather than the outgoing flux at the horizon. On the other hand, we also obtained that the Hawking radiation in the Unruh vacuum comes from the positive outward flux in the near-horizon quantum region of the atmosphere, not right at the horizon. The right temperature became finite everywhere, so that the low energy Hawking particles turned out to be irrelevant to the infinite blueshift. Consequently, the present study shows that the firewall from the infinite Tolman temperature at the horizon and the Hawking radiation from the atmosphere outside the horizon are compatible, if we discard the claim that the Hawking radiation in the Unruh vacuum originates from the infinitely blueshifted outgoing excitations at the horizon.

As for the information loss paradox, the present firewall need not play a role of the entanglement-breaker between two partners of each pair across the horizon created from pair production, but it is due to the infinite blueshift of the influx at the horizon. So, the firewall might be regularized in certain ways in order to save the violation of the equivalence principle at the horizon. Regarding the firewall, it was claimed that there are no event horizons [34], and they are inappropriate to describe the evaporating black hole practically [35]. In addition, it was shown that the quantum back reaction of the geometry renders a star stop collapsing a finite radius larger than its horizon [36, 37]. Recently, it was also claimed that the firewall is due to the limitation of the semiclassically fixed background [38]. In fact, the present study was also performed on such a fixed background with the event horizon. In these regards, it could be speculated that the firewall in the Unruh vacuum might be smoothed out by taking into account the quantum back reaction of the geometry going beyond semiclassical approximations, which remains unsolved.

In connection with the above comment, the authors [38] argued that the firewall paradox manifests as a limitation of semiclassical treatment in that the semiclassical theory possesses 
an unphysically large Fock space built by creation and annihilation operators on a fixed black hole background. The firewall is in essence the divergent quantity in the presence of the event horizon on the fixed background. In these regards, the firewall should be regularized by taking into account appropriate back reaction of geometry, and then I just speculate that the ingoing modes might be a comparable wavelength to that of the outgoing flux. However, the process of information recovery is another issue, whether the nonlocal quantum field theory advocated by Giddings [10] is essential or not; otherwise, the other mechanisms responsible for the information retrieval might be needed.

The final comments are now in order. Firstly, the decomposition of left-right fluxes is based on the classical picture of radiation with a well-defined classical two-velocity written in the form of light-cone coordinates. So, one might wonder whether this geometrical approach of decomposition of fluxes is valid or not. The flux $\left\langle T^{01}\right\rangle$ in an inertial frame was given by Eq. (7), and then it was decomposed into left-right fluxes, which are indeed attributed to the massless property of radiation. If the Hawking particles were massive, then the chiral decomposition of $\left\langle T^{01}\right\rangle$ would be impossible. Moreover, the two-velocity and the normal vector play a role of transformation matrices from the tortoise coordinate system to the locally inertial coordinate system as seen from Eq. (6). Hence, it is valid to treat these vectors as classical objects as long as the background metric is fixed in the present semiclassical approximation. If the back reaction of the geometry were taken into account, then the velocity and the normal vector should be corrected in accord with quantum corrections. Secondly, the typical wavelength of outgoing Hawking modes is comparable to the size of the black hole. The wavelength of negative ingoing modes is also the same order of the critical radius $r_{c}$ at which the two free-fall temperatures become equal. Now, one might wonder how much ingoing modes are expected to overlap with the typical outgoing modes. This issue will be discussed only in freely falling frames such that physical quantities are proper ones, for example, the left-right fluxes mean the proper left-right fluxes as $\mathcal{F}_{L, R}$ rather than the flux $\left\langle T_{ \pm \pm}\right\rangle$defined in the tortoise coordinates, but they are related to each other in the way of $\mathcal{F}_{L, R}=\mp\left\langle T_{ \pm \pm}\right\rangle / g(r)$ from Eq. (4). The thermal temperature is usually interpreted as an absolute value of momentum for a particle. The left temperature from the influx is inversely proportional to the wavelength of ingoing modes. In particular, the wavelength of ingoing modes is the same as that of outgoing modes at $r_{c}$. However, the wavelength of ingoing modes generically depends on a free-fall position critically, while the size of the wavelength 
of outgoing modes is almost comparable to the size of the black hole except for free-fall positions near the horizon as seen from Fig. 1. At the horizon, the influx is divergent at the horizon, and so the left temperature is also divergent, while the outward flux and the right temperature approach zeroes there. It means that the wavelength of ingoing modes in the freely falling frame near the horizon is extremely short in contrast to the very long wavelength of outgoing modes. On the other hand, at the asymptotic infinity, the wavelength of ingoing modes approaches infinity while the wavelength of outgoing modes becomes that of the well-known Hawking modes. Consequently, the wavelength of ingoing modes is highly sensitive to the free-fall positions in contrast to that of outgoing modes. As was discussed earlier, we just speculate that if the divergent ingoing modes could be regulated properly by taking into account the back reaction of the geometry, then they would be finite. Finally, it has been widely believed that a freely falling observer encounters nothing out of the ordinary when crossing the horizon based on the equivalence principle. In fact, there is no reason for a freely falling observer to find the divergent proper energy-momentum tensor since the curvature in the free-fall frame is finite everywhere except the origin. Thus, the finite proper influx saves the violation of the equivalence principle except for the horizon where the firewall is located [3].

\section{ACKNOWLEDGMENTS}

I have benefited from discussions with M. Eune, Y. Gim, and E. J. Son, and especially thank W. Israel for introducing his helpful paper for improvement of the present work.

[1] S. W. Hawking, Particle Creation by Black Holes, Commun. Math. Phys. 43 (1975) 199-220.

[2] L. Susskind, L. Thorlacius and J. Uglum, The Stretched horizon and black hole complementarity, Phys. Rev. D48 (1993) 3743-3761, [hep-th/9306069].

[3] A. Almheiri, D. Marolf, J. Polchinski and J. Sully, Black Holes: Complementarity or Firewalls?, JHEP 02 (2013) 062, [1207.3123].

[4] S. L. Braunstein, S. Pirandola and K. Zyczkowski, Better Late than Never: Information Retrieval from Black Holes, Phys. Rev. Lett. 110 (2013) 101301, [0907.1190]. 
[5] D. N. Page, Information in black hole radiation, Phys. Rev. Lett. 71 (1993) 3743-3746, [hep-th/9306083].

[6] R. Bousso, Complementarity Is Not Enough, Phys. Rev. D87 (2013) 124023, [1207.5192].

[7] Y. Nomura, J. Varela and S. J. Weinberg, Complementarity Endures: No Firewall for an Infalling Observer, JHEP 03 (2013) 059, [1207.6626].

[8] L. Susskind, Singularities, Firewalls, and Complementarity, 1208.3445.

[9] S. Hossenfelder, Comment on the black hole firewall, 1210.5317.

[10] S. B. Giddings, Nonviolent information transfer from black holes: A field theory parametrization, Phys.Rev. D88 (2013) 024018, [1302.2613].

[11] A. Almheiri, D. Marolf, J. Polchinski, D. Stanford and J. Sully, An Apologia for Firewalls, JHEP 1309 (2013) 018, [1304.6483].

[12] J. Hutchinson and D. Stojkovic, Icezones instead of firewalls: extended entanglement beyond the event horizon and unitary evaporation of a black hole, 1307.5861.

[13] B. Freivogel, Energy and Information Near Black Hole Horizons, JCAP 1407 (2014) 041, [1401.5340].

[14] W. G. Unruh, Notes on black hole evaporation, Phys. Rev. D14 (1976) 870.

[15] W. G. Unruh, Dumb holes and the effects of high frequencies on black hole evaporation, gr-qc/9409008.

[16] R. Casadio and L. Mersini-Houghton, Short distance signatures in cosmology: Why not in black holes?, Int. J. Mod. Phys. A19 (2004) 1395-1412, [hep-th/0208050].

[17] W. Israel, Shenanigans at the black hole horizon: pair creation or Boulware accretion?, 1504.02419.

[18] S. B. Giddings, Hawking radiation, the StefanBoltzmann law, and unitarization, Phys. Lett. B754 (2016) 39-42, [1511.08221].

[19] D. N. Page, Particle Emission Rates from a Black Hole: Massless Particles from an Uncharged, Nonrotating Hole, Phys. Rev. D13 (1976) 198-206.

[20] S. B. Giddings, Black hole information, unitarity, and nonlocality, Phys. Rev. D74 (2006) 106005, [hep-th/0605196].

[21] R. C. Tolman, On the Weight of Heat and Thermal Equilibrium in General Relativity, Phys. Rev. 35 (1930) 904-924. 
[22] J. B. Hartle and S. W. Hawking, Path Integral Derivation of Black Hole Radiance, Phys. Rev. D13 (1976) 2188-2203.

[23] W. Israel, Thermo field dynamics of black holes, Phys. Lett. A57 (1976) 107-110.

[24] Y. Gim and W. Kim, A Quantal Tolman Temperature, Eur. Phys. J. C75 (2015) 549, [1508.00312].

[25] S. Deser, M. J. Duff and C. J. Isham, Nonlocal Conformal Anomalies, Nucl. Phys. B111 (1976) 45.

[26] S. M. Christensen and S. A. Fulling, Trace Anomalies and the Hawking Effect, Phys. Rev. D15 (1977) 2088-2104.

[27] R. M. Wald, Gravitation, thermodynamics, and quantum theory, Class. Quant. Grav. 16 (1999) A177-A190, [gr-qc/9901033].

[28] M. Eune, Y. Gim and W. Kim, Something special at the event horizon, Mod. Phys. Lett. A29 (2014) 1450215, [1401.3501].

[29] C. G. Callan, Jr., S. B. Giddings, J. A. Harvey and A. Strominger, Evanescent black holes, Phys. Rev. D45 (1992) 1005-1009, [hep-th/9111056].

[30] D. G. Boulware, Quantum Field Theory in Schwarzschild and Rindler Spaces, Phys. Rev. D11 (1975) 1404.

[31] H. Boschi-Filho and C. P. Natividade, Anomalies in curved space-time at finite temperature, Phys. Rev. D46 (1992) 5458-5466.

[32] M. Visser, Gravitational vacuum polarization. 3: Energy conditions in the $(1+1)$ Schwarzschild space-time, Phys. Rev. D54 (1996) 5123-5128, [gr-qc/9604009].

[33] D. Singleton and S. Wilburn, Hawking radiation, Unruh radiation and the equivalence principle, Phys. Rev. Lett. 107 (2011) 081102, [1102.5564].

[34] S. W. Hawking, Information Preservation and Weather Forecasting for Black Holes, 1401.5761.

[35] M. Visser, Physical observability of horizons, Phys. Rev. D90 (2014) 127502, [1407.7295].

[36] L. Mersini-Houghton, Backreaction of Hawking Radiation on a Gravitationally Collapsing Star I: Black Holes?, Phys. Lett. B738 (2014) 61-67, [1406.1525].

[37] L. Mersini-Houghton and H. P. Pfeiffer, Back-reaction of the Hawking radiation flux on a gravitationally collapsing star II, 1409.1837.

[38] Y. Nomura and N. Salzetta, Why Firewalls Need Not Exist, 1602.07673. 